\title{
Crowd-sensing our Smart Cities: a Platform for Noise Monitoring and Acoustic Urban Planning
}

\author{
Marco Zappatore, Member, IEEE, Antonella Longo, Member, IEEE, and Mario A. Bochicchio
}

\begin{abstract}
Environmental pollution and the corresponding control measurements put in place to tackle it play a significant role in determining the actual quality of life in modern cities. Amongst the several pollutant that have to be faced on a daily basis, urban noise represent one of the most widely known for its already ascertained health-related issues. However, no systematic noise management and control activities are performed in the majority of European cities due to a series of limiting factors (e.g., expensive monitoring equipment, few available technician, scarce awareness of the problem in city managers). The recent advances in the Smart City model, which is being progressively adopted in many cities, nowadays offer multiple possibilities to improve the effectiveness in this area. The Mobile Crowd Sensing paradigm allows collecting data streams from smartphone built-in sensors on large geographical scales at no cost and without involving expert data captors, provided that an adequate IT infrastructure has been implemented to manage properly the gathered measurements. In this paper, we present an improved version of a MCS-based platform, named City Soundscape, which allows exploiting any Android-based device as a portable acoustic monitoring station and that offers city managers an effective and straightforward tool for planning Noise Reduction Interventions (NRIs) within their cities. The platform also now offers a new logical microservices architecture.
\end{abstract}

Index Terms-Acoustic monitoring, microservices architecture, mobile crowd sensing, noise monitoring, smart city, urban planning.

\section{INTRODUCTION}

$\mathrm{T}$ HE recent years have seen a considerable increase in the number of cities aiming at becoming smart by exploiting the continuous advancement in ICT (Information and Communication Technology) solutions and devices. Hundreds of technology-based administrative programmes have been developed worldwide in order to deploy solutions capable of improving citizenship's life quality, enforcing public security, supporting local governmental activities, boosting the economy, modernizing urban infrastructures and creating novel

Manuscript received January 13, 2017; revised May 15, 2017. Date of publication June 1, 2017.

This work is part of the EU funded project "SP4UM" (Grant agr. n. 632853 , sub-grant n. 021), within the "frontierCities" FIWARE accelerator.

Authors are with the Department of Innovation Engineering, University of Salento, Lecce, 73100 Italy (e-mails: \{marcosalvatore.zappatore, antonella.longo, mario.bochicchio\}@unisalento.it).

Digital Object Identifier (DOI): 10.24138/jcomss.v13i2.373 market and financial opportunities. Several examples of effective smart cities initiatives have been showcased so far worldwide, ranging from small cities [1] to huge metropolitan areas [2].

These initiatives target either the retro-fitting of already existing cities (as in the majority of the cases) or the creation of new cities from scratch (as for the new city of Songdo in South Korea) [3]).

Modern cities are tasked to face numerous challenges: environmental risks, demographic shifts, population growth, natural disaster response, constantly-increasing power demands, disaffection of citizens towards policy makers and so on. Therefore, a smart city is more and more seen as a dynamic and complex system where modern technologies aim not only at informing the citizenship more efficiently but also at fostering a more active engagement of the citizenship itself into the collective management of the city.

Other core objectives can be recognized as well, such as: improving city services efficiency, increasing city control, raising citizenship security, favoring economic development. However, the smartness of the financial, economic, infrastructural, societal, environmental and productive contexts can be achieved only by allowing all the involved entities to work and live in an integrated and coherent way, thanks to several technological drivers:

1) Data Gathering

Easy data-collection across the city and proper dataintegration from heterogeneous data sources allow achieving a detailed and measurable live knowledge of the current city status.

2) Data Access

Effective (and possibly open) access and visualization strategies for collected and processed data allow citizens, city managers and service providers fulfilling their tasks more efficiently and also enabling a continual sharing of significant information.

3) Data Analytics

The availability of analytics and decision-making systems supports city managers and citizens in applying the most appropriate actions and interventions to be delivered across their city.

While several activities in the area of the Internet of Things and of embedded computing have already been exploited in the 
context of Smart Cities, especially for healthcare-related sensors [4], [5] and ICT platforms [6], other novel promising paradigms enabling horizontal and vertical Smart City-oriented solutions are emerging as well. Smartphones and mobile devices, indeed, have the potential to address civic challenges very effectively, from disaster response to public health and safety, from location-oriented service provisioning to social integrations of disadvantaged people, from service access and delivery improvement to professional and educational training. This is not only due to the progressive governmental shifts towards online and mobile services but also to the sensing capabilities of modern smartphones. Therefore, mobiles can be dynamically scattered across huge areas with heterogeneous sensing purposes and they can acquire contextual awareness opportunistically from the surrounding environment [7] Especially in urban contexts, citizens can receive, provide and share information about specific situations occurring around them thanks to mobiles, thus enabling the crowd monitoring [8]. This approach is nowadays widely known as Mobile Crowd Sensing (MCS) [9]. MCS-related opportunities are directly disclosed by the capacity of collecting location-based data provided by mobile devices in an unprecedented way. Mobile device allow to relate and correlate geo-referenced sensor streams to physical and social data. As a consequence, citizens can acquire greater knowledge on their urban landscape and city managers can achieve better knowledge on people's perception of their city, thus tailoring civic policies more effectively to the real needs of the population.

The main research aim of this paper is to address the domain of mobile sensing as a key enabler for developing smart citizens and smart administrators in our cities. More specifically, we have chosen to examine the acoustic monitoring area as it is considered one of the five most relevant causes of public concerns in European cities due to its implications for citizens' quality of life. We have addressed the following research purposes by developing a technological platform, named City Soundscape, aiming at:

1) allowing low-cost, large-scale, sufficiently-accurate acoustic monitoring campaigns, thanks to a dedicated, Android-based, mobile app.

2) Involving citizens as direct data captors (thanks to the mobile app mentioned above) and offering them a series of straightforward educational opportunities for becoming more aware in the acoustic domain.

3) Offering city managers suitable tools (i.e., a Web app providing noise measurement georeferencing and suggesting real-time evaluation of noise reduction alternatives) for profitably planning the acoustic map of their cities via proper noise abatement interventions.

City Soundscape extends both the logical architecture and the implemented functionalities of the platform presented by the authors at SpliTech2016 conference [10], in order to improve its effectiveness and its usability as a suitable tool for assisting city managers and citizens in assessing the levels of acoustic pollution within their urban contexts.

Several theoretical and methodological novelties have been introduced in comparison to the platform discussed in [10], as its current version derives now more rigorously from the foundational requirements of the MCS paradigm.

Amongst the implementation novelties that have been introduced, the most important one pertains to the platform logical architecture, whose early design choices have been deeply revised and enhanced according to the MicroServices Architectural (MSA) approach. In addition, the proposed Web app - which is tailored to city managers' needs and capable of suggesting proper noise abatement/reduction interventions on the bases of the noise measurements collected by citizens' smartphones - has been completely revised after a series of trial tests performed with several city managers in the geographical are where the platform prototype has been deployed.

The paper is organized as follows: Section II offers an up-todate review of smart city-related definitions and conceptions from the most recent works in scientific and corporate literature. The adopted MCS paradigm and its foundational principles are discussed in Section III, whilst Section IV presents and evaluates the current state of the art in the MCS-based acoustic and noise monitoring scenario. The urban noise research domain is addressed in Section $\mathrm{V}$, by highlighting its correlation with public health issues and on the regulatory aspects (European and Italian ones). Section VI presents the proposed platform, by paying specific attention to data modelling aspects and by presenting the adopted new MicroServices Architecture (MSA) of the City Soundscape platform. Section VII presents the functional validation of the two main platform components, namely the data-collecting mobile app and the acoustic urban planning web-based app made available to city managers. Conclusions are drawn in Section VIII.

\section{SMart Cities AND Mobile DeVICES}

Smart cities represent both a concept and a model for urban realities, where novel information and communication technologies (ICTs) can be profitably applied to facilitate the design, enactment and management of smart services within urban scenarios. Several innovative IT opportunities are amenable to be leveraged: Internet of Things, Cloud Computing, Big Data, mobile device pervasiveness, information integration and management. According to the BSI PAS 180 [11] group, smart cities are based upon the "effective integration of physical, digital, and human systems in the built environment to deliver a sustainable, prosperous and inclusive future for its citizens". In the same way, the ITU-T Focus Group [12] has proposed the following one: "a smart city is an innovative city that uses ICTs [...] to improve quality of life, efficiency of urban operations and services, and competitiveness, while ensuring that it meets the needs of present and future generations with respect to economic, social and environmental aspects"

The academic and corporate literatures have described the evolution of such concepts and initiatives starting from the "cybernetic and wired cities" [13] envisioned since the early $70 \mathrm{~s}$ towards the modern concept of smart cities mentioned above. This has contributed significantly in identifying three major categories to which a smart city can belong, according to Kitchin [13]. 
In the first category, cities are considered smart when novel ICT solutions are employed for changing configuration and management of urban infrastructures and services [14]. This is normally achieved by deploying amounts of networked digital devices (e.g., wireless sensor networks, smart meters, digital CCTVs, etc.) throughout a city and by complementing them with any other device capable of generating data streams and, at the same time, presenting information such as mobile devices (e.g., smartphones, tablets, smartwatches, etc.). Therefore, not only urban areas can be dynamically monitored and reactively managed but also several planning interventions can be considered and evaluated by taking advantage from the datadriven, networked technologies available in the city.

A second way of thinking about smart cities is to consider in such a way a city when its governing authorities rely on strategic ICT resources such as e-government, e-democracy, eparticipation and open data for achieving considerable improvements in education, citizenship participation, city management and sustainability, economy and innovation [15]. In other words, this way of perceiving smart cities is based upon the idea that technological advancements have to be leveraged in order to foster smart citizens who, on their turn, can trigger a series of actions leading to the "smartification" of their cities.

Alternatively, a smart city can be also conceived as an urban community promoting a smart society. According to this third typology, ICT solutions should be adopted for creating new opportunities of social innovation and inclusion, transparent and participated government, "hactivism" (i.e., situations where citizens are involved in civic hacking and hackathons) [14] Accordingly, a smart city behaves as a citizen-centered community rooted on participatory urban planning, fruition of freely accessible ICT solutions, open usage of digital platforms and data.

Mobiles, therefore, represent a unifying element of the smart city conceptions enlisted so far. First, mobiles allow defining and provisioning innovative services capable of managing contextual information and suitable to interact with user's social and physical situations. Second, mobiles represent a promising solution for engaging people in collaborative, participatory environmental sensing experiences. This may effectively promote wide participatory contributions from citizens, yearning of life quality improvement, as well as positive behavioural changes in citizenship about environmental sustainability. Additionally, this makes possible to harvest large and heterogeneous amounts of information from citizens, describing their continuously evolving urban environments. Such data can be forwarded to city managers, thus allowing them to have better awareness of the potential issues affecting their municipalities, without relevant additional costs. Finally, mobiles can enlarge the scope of traditional monitoring campaigns significantly, so that the expensive deployment and maintenance of professional metering equipment can be spared for ad-hoc interventions only in those city areas where the higher pollution levels are detected.

All these aspects represent the core MCS features, as described in Section III.

\section{MobiLe Crowd-Sensing}

\section{A. Core Features}

Recent market analyses reveal a worldwide penetration rate of $97 \%$ (129\% in Western Europe) for mobiles in 2016 [16] and the envisioned trend for 2020 is even more promising, with 9.2bn mobile subscriptions worldwide. Such a diffusion is mainly facilitated by high data rates, reliable coverage, high Quality of Service, extreme portability, data plans and monthly bills less expensive than fixed-broadband plans. By using smartphones anywhere and anytime, a true device pervasiveness can be achieved. In addition, mobiles exhibits powerful and sufficiently-accurate built-in sensors. The MCS paradigm [17] was proposed to leverage this favorable context, by defining how data collection should be performed directly from mobiles. MCS also allows implementing innovative services for managing contextual information and interacting with user's social and physical situations. Moreover, it makes possible to harvest large and heterogeneous amounts of information from citizens, regarding their continuously evolving urban environments, thus representing a promising way for city managers to acquire better awareness on their municipalities without additional costs.

\section{B. Key Design Requirements for MCS-based Solutions}

In order to implement a MCS-based app profitably, several requirements have to be matched. The authors of [18], by starting from their aim of developing an app for landscape an urban planning, provide a list of needed features a MCS-app must exhibit. Therefore, we have elicited a subset of such requirements, as enlisted in the following, in order to guide our research work:

1) Situated data creation: the app has to provide location-based and geo-referenced data.

2) Sensing typology: users should be allowed selecting whether actively monitoring and contributing data (i.e., participatory sensing) or let the app collect autonomously data in background.

3) Data resolution: data should be collected with high-spatial resolution and in a wide variety of scenarios.

4) Data reliability: once gathered, sensing data have to be processed for determining their accuracy and, if needed, for applying proper data manipulation and management operations.

5) Effectiveness: the app has to provide effective data and information visualization and representation.

6) Time and space continuity: no specific time-related or spacerelated usage limitations should hinder the app usage.

7) Learning opportunities: the app should also exhibit learning elements in order to improve users' awareness on specific topics and/or domains.

8) User involvement: the app should be capable of motivating citizens in participating and of engaging them in actively contributing to the general improvement of their city.

All these requirements have been considered for the design and implementation of the MCS component of the City Soundscape platform, as described from Section VI onwards. 


\section{MCS Applications: State of the Art}

The initial application area of mobile sensing was represented by personalized healthcare and wellness monitoring, as the mobile devices was primarily interpreted as a personal sensing tool capable of helping in the assessment of health parameters thanks to smartphone-pluggable or Bluetooth-enabled external devices (e.g., blood pressure monitors, body analysis scanners, pulse oximeters, glucomonitors, etc.). Despite this trend is still generating a considerable stream of applications [19], other sectors are gaining momentum and benefitting from the MCS approach.

Urban contexts belong to this category, as they are nowadays very promising scenarios for MCS applications. Four core monitoring areas can be identified:

1) Transportation and mobility. Typical examples are: road condition detection [20], traffic flow monitoring [21] and parking monitoring [22].

2) Environment. Quite all the relevant pollutants can be addressed by proper MCS-based monitoring solutions, such as air pollution control [23], water quality monitoring [24], electromagnetic field assessment [25], [26] and radiation detection [27]. Noise monitoring is also present with several MCS applications, which will be discussed in Section IV in more details.

3) Natural disaster [28] and emergency management [29].

4) Large-scale events (e.g., festivals, sport events, etc.) [30].

\section{Mobile Crowd-Sensing FOR ACOUstic Monitoring}

The acoustic monitoring domain has been addressed recently by several MCS applications. However, it would be more proper to consider these applications as mobile-sensing solutions for personal usage, since they are mainly aimed at assessing noise levels surrounding the smartphone user by mimicking the core functionalities of Sound Level Meters (SLMs). Indeed, a common feature in acoustic-monitoring mobile apps is their UI, which typically reproduces the gauges and indicators of a SLM and provides numerical insights on the core noise exposure quantifiers (i.e., real-time acoustic amplitude and derived time-averages with different timelengths). Several mobile apps offering these functionalities are currently available in both Google Play and Apple Store, such as [31], [32] and [33]. The main drawback in these apps is that they do not provide measurement aggregation on a geographical/temporal basis. This means they do not allow to examine, on a map, measurements points collected by different users within a specific temporal window, since they only offer live acoustic evaluation on the single mobile device they are installed into and local measurement history capabilities.

Recently, some research projects have addressed also urban noise mapping, thus entering into the actual domain of MCS. One of the very first applications in this sense was represented by "Ear-Phone" [34], where smartphones were used to predict outdoor sound levels. Similarly, in "2Loud?" [35], iPhones were used to assess nocturnal noise within buildings near highways in Australia. An advanced citizen-based acoustic and weather observation project, named CITI-SENSE [36] has been recently proposed in order to involve citizens in "environmental governance" and to influence decision-makers' actions. Other recent projects aim at geo-referencing acoustic measurements collected via smartphones in urban environments, such as [37] in Antwerp and [38] in Paris. However, these initiatives still exhibit some limitations if evaluated with respect to a true MCS approach. The City City Soundscape, which will be thoroughly discussed in Section VI, aims at tackling these aspects and enhancing the potentials of MCS-based acoustic monitoring. In the remaining part of this Section, the main drawbacks of the solutions mentioned so far will be enlisted and the corresponding City Soundscape functionality will be introduced as well.

First of all, a MCS-based application has to involve its users in several ways: whilst in [34] and [35] users are only involved as data collectors, the most recent trends in research suggest a wider and diversified user's engagement [39], [40]. Scientific learning opportunities, in-app educational contents and elements triggering awareness on environmental sustainability should be considered as additional features of MCS apps. For such reasons, the mobile app provided by the City Soundscape platform not only enables acoustic data collection, but it also offers its users a set of support materials describing the monitored physical quantities, the correct measurement methodologies, the current acoustic monitoring regulations, etc.

Similarly, a more and more requested added value in MCS solutions is represented by the possibility of using the collected datasets for driving proper improvements in life quality conditions. These aims can be achieved by helping authorities and city managers in better assessing the status of their urban contexts, thus fostering one of the core targets of Smart Cities (i.e., leveraging citizens' participation in order to make the city a better place [41]). In [36]-[38], no specific functionality is tailored to city managers for allowing them to improve urban life quality and helping them during the decision-making process. Therefore, City Soundscape also offers a dedicated Web application which has decision-support capabilities for suggesting city managers how to reduce noise impacts on a given area of their city.

Moreover, several current research works focus only on specific aspects in the MCS context, such as in [36], where the societal impact of the project represents one of the core research aspects, or in [38], where the adopted middleware for managing sensor data is the core research topic. All these elements of improvement are proposed within the City Soundscape platform described in Section VI.

\section{URBAN NOISE}

\section{A. Acoustic Emissions, Sources and Health-related Issues}

The majority of Europeans believes that noise (72\%) represents the fifth most significant problem within their cities after air pollution $(81 \%)$, road congestion $(76 \%)$, travelling costs $(74 \%)$ and accidents $(73 \%)$. The noise pollution concern reaches even higher values in Italy $(83 \%)$, Bulgaria $(85 \%)$, Greece $(87 \%)$ and Malta (92\%). If examined from a sociodemographic point of view, the problem is less considered by 
students (66\%) and much more by managers (76\%) [42]. However, still a low number of cities and administrations implements noise-control policies against potential health risks despite the ascertained citizens' concerns about noise pollution issues. Southern European countries reveal a scarcity in noise interventions performed by their municipalities (e.g., just around 1\% in Italy [43]) and that national and regional acoustic classification plans still have to be applied on a large scale (only $53 \%$ of municipalities in Italy has activated such plans [43]).

On the one hand, several factors hinder the implement of stable noise monitoring and mapping policies by local authorities: high equipment costs (e.g., professional fixed monitoring station buying costs: up to $25 \mathrm{k} €$, rental costs: up to $3 \mathrm{k} € /$ month; portable professional SLM: up to $5 \mathrm{k} €$ ), scarcity of skilled personnel and lack of environmental awareness.

On the other hand, modern cities hosts multiple sound sources, which generates acoustic emissions exhibiting different characteristics in frequency and time. A widely adopted categorization partitions such emissions into three categories.

1) Impulsive acoustic emission: it is due to short-duration pulses having random amplitude and random short duration (e.g., <1s). Typical impulsive sources are hammering noises and gunfire.

2) Transient acoustic emission: it consists of sound pulses having longer duration or relatively short pulses followed by decaying low frequency oscillations. Vehicle pass-by, aircraft flyover and crowd chanting after an event are the most common transient sources.

3) Continuous acoustic emission: it exhibits stable conditions over a relatively long temporal period. These emissions are typically generated by industrial plants working for several hours without any interruption

The most relevant source for acoustic emissions in urban contexts is represented by vehicular traffic. It is generated by multiple components (e.g., engine and transmission, tires, aerodynamic, braking systems, vehicle-mounted devices, etc.) and it also strongly varies depending on vehicle typology, speed and age [44].

Road traffic emissions are typically sided by the emissions from roadwork (e.g., asphalting and utility companies) and construction sites, which typically exhibit long time durations.

Leisure time activities such as concerts and festivals represent another significant source of noise, especially when rock and pop music are played. Recent studies highlighted how years of exposure to loud music played at discotheques and during concerts may induce irreversible noise-induced hearing loss in both ears of at least $10 \mathrm{~dB}$ at $3 \mathrm{kHz}$. Recent tests activities performed during music festivals highlighted how the individual sound exposure per evening varied between 90 and $115 \mathrm{~dB}(\mathrm{~A})$, with an average exposure of $100 \mathrm{~dB}(\mathrm{~A})$ and prolonged peaks of $110 \mathrm{~dB}(\mathrm{~A})$ [45].

The noise generated by airplanes (e.g., during take-off and landing phases) and airport installations (e.g., during airport activities) is another major source of disturbance, especially when airports are in close proximity to cities as in the majority of the European cities [46].
TABLE I

Acoustic Classes and Corresponding Threshold Values ACCORDing to ItALian Regulations [54], [53]

\begin{tabular}{|c|c|c|c|c|c|c|}
\hline \multirow{2}{*}{ Acoustic Class } & \multicolumn{2}{|c|}{ Limit } & \multicolumn{2}{|c|}{ Quality } & \multicolumn{2}{|c|}{ Attention } \\
\hline & day & night & day & night & day & night \\
\hline C1. Protected & 45 & 35 & 47 & 37 & 50 & 40 \\
\hline C2. Residential & 50 & 40 & 52 & 42 & 55 & 45 \\
\hline $\begin{array}{l}\text { C3. Mixed } \\
\text { C4. Intense }\end{array}$ & 55 & 45 & 57 & 47 & 60 & 50 \\
\hline $\begin{array}{l}\text { human } \\
\text { activities }\end{array}$ & 60 & 50 & 62 & 52 & 65 & 55 \\
\hline $\begin{array}{l}\text { C5. Mainly } \\
\text { industrial }\end{array}$ & 65 & 55 & 67 & 57 & 70 & 60 \\
\hline $\begin{array}{l}\text { C6. Exclusively } \\
\text { industrial }\end{array}$ & 65 & 55 & 70 & 70 & 70 & 70 \\
\hline
\end{tabular}

From a health-related perspective, the necessity of proper noise monitoring activities is enforced also by the correlations between health and acoustic emissions [47] as outlined by several epidemiological analyses. Even if depending on multiple causes and on individual predispositions, environmental noise sources can determine acute effects (e.g., temporary noise-induced hearing loss), chronic effects (e.g., sleep disturbance and fragmentation, reduced productivity) and long-term risks (e.g., cardiovascular diseases, permanently noise-induced hearing loss).

Moreover, especially in urban scenarios, noise can cause the so-called noise annoyance [48], which stands for a series of socio-behavioural changes and overall discontent in citizens residing in noisy areas that may determine additional effects (e.g., increased drug consumption, increased number of accidents).

Acoustic emissions also affect more heavily specific categories of subjects or people exhibiting additional health risks: for instance, children living in noisy contexts or attending schools located in dense urban areas show poor performances, stress, decreased learning rates, misbehaviour, concentration deficits, hyperactivity and scarce reading comprehension [49]. The chronically ill and the elderly are two other population categories especially vulnerable to noise-related diseases.

\section{B. Current Noise Monitoring Regulations}

The most important regulatory contribution pertaining to noise pollution at European level is provided by the Directive 2002/49/EC relating to the assessment and management of environmental noise (the so-called END, Environmental Noise Directive) [50]. The END main purpose is to trigger the necessary actions both at Member State and EU level for:1) determining the exposure to environmental noise; 2) ensuring that information on environmental noise and its related effects reaches the public; 3) preventing and reducing environmental noise when and where needed. The END requires each Member State to prepare and publish with a 5-year frequency, noise maps and noise management action plans (which has to be developed by consulting the concerned citizenship) for cities having more than $100 \mathrm{k}$ inhabitants.

The END has been examined in 2015 and 2016 by the EU commission in the framework of its REFIT programme, aiming evaluating the "regulatory fitness" of EU laws (i.e., "identifying actions to make EU laws simpler, more efficient and effective") 
[51]. After such evaluation, the END has been evaluated as "highly relevant for EU policy-making as noise pollution still constitutes a major environmental problem in Europe" but its effectiveness has been hindered "due to the delays in adopting common assessment methodologies" by Member States. Moreover, "noise population exposure data was so far not used for legislation on noise at source". These aspects, in conjunction with the absence in the END of any limit or target values for environmental noise monitoring make clear that the management of environmental noise in Europe is far from being effectively tackled.

Therefore, Member State legislative actions have to manage such issues at their discretion. In such a regulatory scenario, the Italian law is based upon the adoption of two main noise exposure quantifiers. The first one is the Sound Pressure Level $(S P L)$ in units of $d B(A)$ [52], which allows measuring the dependence of perceived loudness w.r.t. frequency. Since sounds are typically fluctuating (i.e., they vary in time and have different durations) and since $S P L$ is an instantaneous measurement instead, the Equivalent Sound Level $L_{E Q(T)}$ is preferred [52] as the reference exposure descriptor in noise regulations and guidelines. It measures, in $d B(A)$, the steady sound level conveying the same sound energy of the actual time-varying noise source in a given place during a given time window $T$ (where $T$ typically ranges from 30 s to $24 \mathrm{~h}$ ). In a more simplified explanation, $L_{E Q(T)}$ averages the $S P L$ values measured during $T$, thus smoothing spikes and outliers.

Italian noise regulations [53] classify urban areas into six acoustic classes depending on their main usage and building typologies. As reported in Table I, different threshold $L_{E Q(T)}$ values are provided for each of those classes. In addition, these thresholds are also expressed w.r.t.: time of the day (diurnal: 6a.m. - 10p.m.; nocturnal: 10p.m. - 6 a.m.); sensor position w.r.t. the noise source (insertion values: if near the source; emission values: if far from the source); road type (w.r.t. vehicle capacity and speed) and age (novel or already existing roads) [54], [55].

The Italian laws adopt a precautionary approach, so that the law thresholds that cannot be trespassed (i.e., limit values) are always below the noise emission values representing a lower risk or a potential risk for human health (i.e., quality values and attention values, respectively). As a reference, it could be useful to consider that in urban contexts typical noise values at $15 \mathrm{~m}$ from the observer are: heavy truck $(90 \mathrm{~dB}(\mathrm{~A}))$; congested city road $(80 \mathrm{~dB}(\mathrm{~A}))$; light car traffic $(60 \mathrm{~dB}(\mathrm{~A}))$.

Therefore, the Italian law determines and provides the thresholds to be abided by, the noise monitoring and controlling procedures, the noise abatement and reclamation techniques. The brief regulatory and normative overview sketched so far highlights how significant for smart cities can be the impact of solutions allowing 1) people to become better aware of noise pollution within their cities and 2) city managers to become more supported in their noise management and reduction actions. The MCS-based platform proposed in this research work exactly aims at this direction.

\section{The City SOUNDSCAPE PlatForm}

\section{A. Overview}

The City Soundscape platform embeds several components in order to meet the requirements from multiple user categories. For city managers, we have developed a decision support system suggesting how to reduce noise levels and where regulatory thresholds are exceeded. For mobile users, we have developed an Android-based mobile app allowing them not only to collect measurements but also to learn about noise metering and acoustic principles directly on their devices.

Before introducing the design and modelling aspects (in the following Subsections) and the platform validation (in Section VII), it is important to summarize the intended usability of the proposed platform, as well as its capability of managing the MCS-related aspects that have been described and discussed so far. City Soundscape is a cloud-based platform that manages acoustic monitoring data collected by mobile devices for generating large-scale, geo-referenced datasets of measurements in order to help city managers in improving the life quality of their cities.

The platform is operative and available online for users (for the description and the available links, see Section VII). Mobile users can download the acoustic monitoring app from Google Play for participatory collecting acoustic measurements (or simply by letting the app collects measurements in background). As a measurement session ends, users can send their data to the central system (and optionally enrich them with comments). Once collected centrally, measurements are evaluated depending on their data quality and data source reliability. Then, measurements are aggregated both temporally and spatially in order to create geo-referenced datasets of acoustic measurements. The datasets are then placed as additional layers on digital maps provided by OpenStreetMap. The geo-referenced datasets are accessible within a dedicated Web application that offers rich visualization options to citizens and additional capabilities for evaluating the best suitable noise reduction/abatement options to city managers. This second category of users is indeed supplied with a decision-support system that enables the evaluation of the amount of achievable noise reduction.

The evaluation is achieved by simulating the effect of applying noise reduction interventions in a specific geographical area, given an acoustic observation dataset in a specific time-window. The noise reduction interventions can be picked from a set of alternatives (e.g, speed bumps, noise barriers, speed enforcement limitations, etc.) that can be applied by local administrators according to current Italian and European laws and regulations.

\section{B. Data Modelling}

When dealing with mobile-generated data streams, data modeling and data management aspects are crucial, as such data streams need to be collected, cleansed and transformed, aggregated and stored in order to make them available for final users. These steps can be tackled very effectively in a Data Warehouse (DWH) approach [56], where data processing is 
performed along an Extraction-Transformation-Loading (ETL) pipeline. Since the DWH approach refers to multidimensional data model, it is very suitable to the management of sensor data. When dealing with DWH applications, a widely-used data modeling technique is given by the Dimensional Fact Model (DFM) [56], which is a conceptual model characterized by a high graphical expressivity, whose clarity allows representing concepts in a straightforward way, thus easing the comprehension of the multidimensional analyses that can be performed on data.

The core element in a DFM is called a fact: it represents any concept relevant to decision-making processes and which evolves in time. Fact attributes describe the fact qualitatively, whilst fact measures provide its qualitative description in terms of numerical properties or relevant calculations.

Being a multidimensional entity, a fact can be analyzed along different coordinates (i.e., dimensions), which enlist several dimensional attributes per each, organized into directed trees departing from the fact.

Dimensional attributes qualify the finite domain of their dimension according to different degrees of granularity (e.g., the temporal dimension can vary from seconds to days, weeks, months; a product is described by its name, series, brand, etc.). The DFM notation as well as the adopted modeling choices are described in Fig.1. This figure depicts the fact "noise measurements", represented as a box with rounded corners. Its fact measures, enlisted within the box, are $S P L$ and maximum/minimum/average $L_{E Q(T)}$.

The fact branches represent its dimensions. Fig. 1 depicts the following ones: time (both timestamp and date/month/year); geographical position (latitude, longitude, town, province, region, country); sensor type (external or embedded); device type (model and brand); measurement type; outlier condition (i.e., when a measurement point significantly differs from the majority of the collected measurements).

We have also considered the possibility for a user to enrich the measurements coming from its smartphone by adding personal comments and opinions, in order to better describe the measurement location as well as the user's perception of the monitored acoustic events. Since user's comments are not mandatory, the corresponding dimension is optional (it is indicated in Fig.1 as the branch having a normal segment crossing it). By considering this dimension, we can model all the opinions a user can have about the undergoing measurement session. First of all, an acoustic source can be considered unique or not, depending on whether the mobile user perceived there is only a predominant acoustic source around her/him or multiple ones. The other available annotations are: source type (i.e., natural or artificial), location type (i.e., indoor or outdoor), annoyance (i.e., annoying or not annoying), nuisance (i.e., how much the acoustic source is deemed noisy by the user) and distance from the observer (i.e., very close, close, quite distant).

In Fig.1, the dimensional attributes for each dimension are represented as circles connected by lines to the fact, whilst the dimension is the root circle of the corresponding branch.

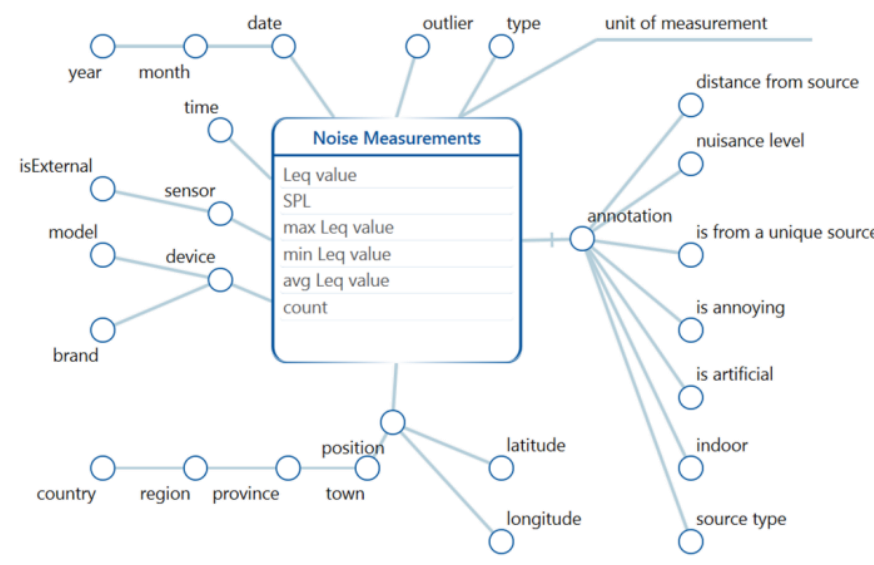

Fig. 1. Dimensional Fact Model (DFM) for the fact "Noise Measurements".

\section{Logical Architecture}

We propose an Android-based application to collect noise measurements from built-in mobile microphones; its interface mimics a professional SLM and allows users to collect peak, average and current values of $S P L$ and $L_{E Q(T)}$ on customizable temporal windows, as required by EU and Italian noise regulations.

Measurements are stored locally (short-term history) and sent to the applications hosted on a server farm for data aggregation (both in time and in space) and filtering. The data brokering functionality is achieved by using Orion [57], a Generic Enabler (GE) from FIWARE middleware [58] that offers publishing/subscribing operations on collected data. Data are persisted on a MongoDB instance, a documental NoSQL DBMS. Additional tasks are performed on server side, such as correlation between annotated and opportunistic measurements or between measurements and vehicular traffic flows. Moreover, the server also hosts a Web application for supporting city managers in managing noise pollution within their cities. Users are allowed to visualize the measurement history and select amongst a list of several Noise Reduction Interventions (NRIs).

From an architectural point of view (Fig. 2), the data layer hosts the non-persistent relational data storage solution for mobile-hosted sensor data, the NoSQL component for persistent storage and a persistent relational DB for noise regulations and guidelines. The context-brokering and data integration layer allows managing data coming from different sensors and offers data integration, filtering and reporting functionalities thanks to Pentaho CE [59], a freeware ETL application. In the same layer is also placed the decision support system for suggesting city managers proper noise abatement interventions depending on the collected measurements.

The data presentation layer hosts a Web app for accessing data reporting and integration results as well as presenting users the suggestion from the decision support system. 


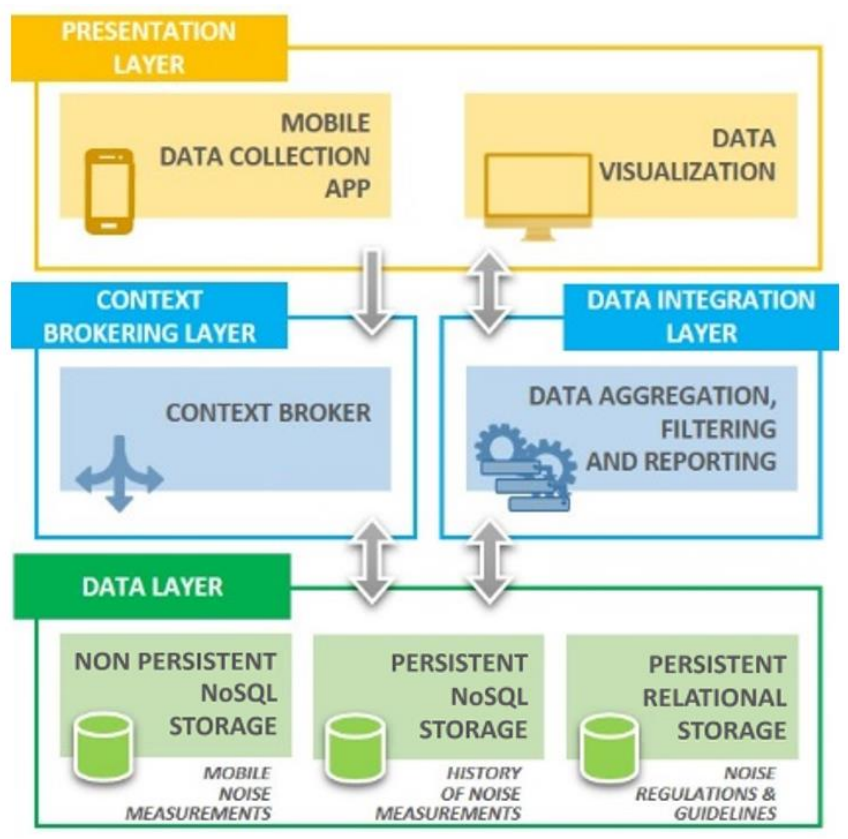

Fig. 2. Platform logical architecture.

\section{Microservices-based Architecture}

Traditional monolithic platform architectures have proven to be very effective in a plethora of scenarios but several issues still exist in terms of deployment and maintenance complexities, scale of downtime events, scalability. In order to overcome these issues, the new MSA architectural style has been proposed [60] in the recent years, aiming at splitting large monolithic applications into collections of microservices having limited and much more focused goals on each own but still acting as a single distributed system.

In MSAs, each functional element can be ideally put into a separate microservice that can be deployed on a different server and no centralized control mechanisms are needed [61]. Each microservice runs its own internal processes and communicates with the other microservices through lightweight protocols, such as RESTful APIs.

Several advantages can be achieved: each microservice has its own capabilities, so that different microservices can be implemented with different business logics, different programming languages and different storage solutions even if concurring to the achievement of the same final purpose.

Moreover, if scaling is needed, since MSAs are loosely coupled, each separate microservice can be scaled and replicated selectively across multiple servers. The same approach also holds when changes or maintenance procedures are needed. Therefore, MSAs allow building scalable, onpremises and cloud-based distributed applications.

It is, however, necessary to apply some careful implementation strategies, such as detailed logging and monitoring of each microservice, efficient event management mechanisms, service decoupling techniques as well as considering dedicated APIs for each microservice and having dedicated development teams for specific application capabilities.

Therefore, the platform logical architecture described in
Section VI.C and depicted in Fig.2 as it was presented in [10], has been now revised and split into a MSA. We have developed 10 microservices, partitioned into three categories.

The new MSA is represented in Fig.3.

The three categories are dedicated to data management in general, noise management in particular and backend procedures. The detailed list of the microservices contained in each category now follows:

1. Data management microservices: this category comprises 7 microservices that are in charge of loading incoming data and of performing the ETL pipeline. More in details, the microservices belonging to this category are:

a. Orion manager: this microservice manages the access to Orion, the Context Broker architectural component managing data incoming from the data layer according to a publish/subscribe mechanism

b. MongoDB \#ORION: this microservice manages the instance of MongoDB dedicated to storing data from/to Orion.

c. Cygnus manager: this microservice manages the communication between Orion and Cygnus, where Cygnus, on its turn, is the application behaving as an interface between the context broker and the data persistence layer according to the FIWARE framework architecture.

d. MongoDB \#CYGNUS: this microservice manages the instance of MongoDB that stores data from/to Cygnus.

e. ETL manager: this microservice manages all the jobs enlisted within the ETL pipelines

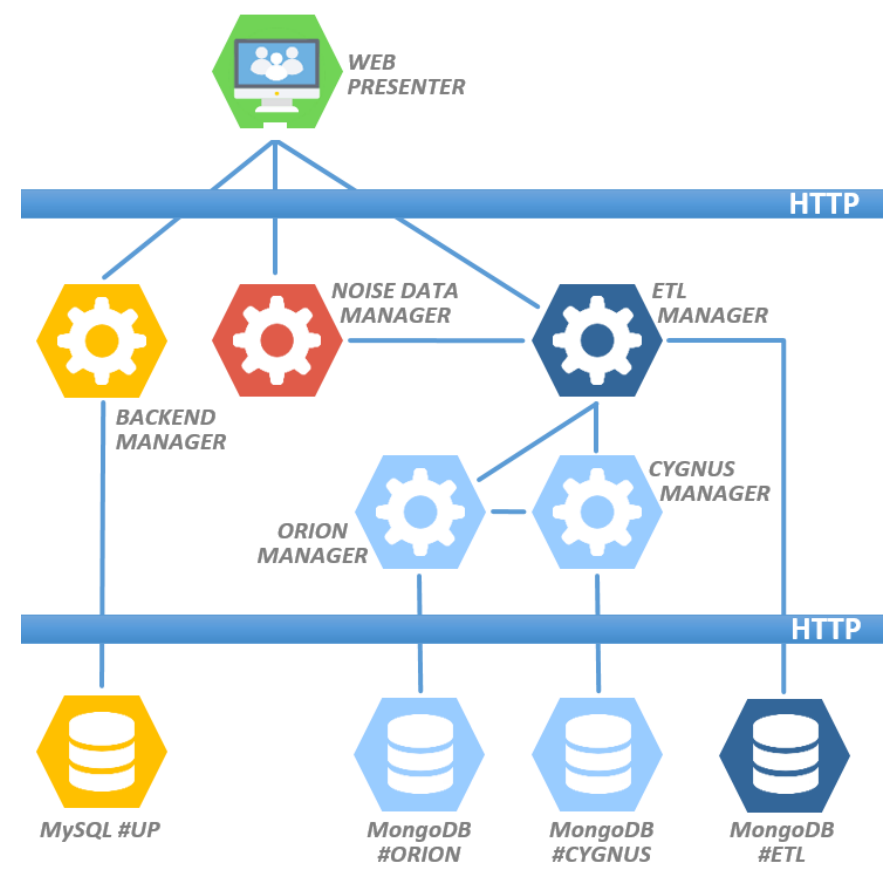

Fig. 3. Platform microservices architecture. 
needed for processing sensor data.

f. MongoDB \#ETL: this microservice manages the MongoDB instance dedicated to storing the cleansed and reconciled data outputted by the ETL pipelines.

g. MYSQL \#UP: this microservice manages user data sent by the backend microservice.

2. Noise management microservices: this category enlists two microservices. The first one (Noise Data Manager) is responsible for the web service that presents noise data and the second one (Web Presenter) manages the Web interface of our Web application.

3. Backend microservices: this category comprises the microservice (Backend Manager) that manages user profiles.

A significant advantage provided by MSAs is represented by its selective scalability. Let us consider our platform: at the moment of writing this paper, the platform engages users in acoustic monitoring. In order to extend the scope of the platform to other typologies of environmental monitoring, it would be enough to add only a set of microservices managing the new parameters to be monitored (e.g., air or water pollutants) instead of upgrading the entire platform.

\section{PLATFORM VALIDATION}

\section{A. Measurement Collection Phase}

According to [62], "noise pollution is a spatially-dependent phenomenon": the analysis of its impacts on the population and the ecosystem should benefit from the usage of Geographic Information Systems (GIS). For this reason, we decided that MCS alone is not sufficient for bringing significant advantages to city managers. Even if MCS plays a pivotal role in collecting measurements easily and engaging users directly in monitoring (thus increasing their awareness on environmental issues challenging their cities), proper geo-referencing solutions are also needed. Therefore, we have developed in [10] a mobile app for measuring acoustic pollution levels by simply exploiting the built-in microphones of any Android-based mobile device. In [10] we have also developed a Web app for accessing measurement history and receiving suggestions on how to reduce noise. This Web app has been totally revised and in Section VII.C it will be described in detail. The platform prototype has been tested in the city of Brindisi, (Apulia administrative region, Southern Italy) by involving local authorities and 7 middle/high schools (19 classes, nearly 300 students and nearly 20 teachers) in order to benefit from a large pool of mobile data captors. Users can download (for free) our data-collection app from the online Google Play store by simply searching for its commercial name "City Soundscape" [63]. Once installed, users can start measuring without plugging into their smartphone any external sensing equipment. More than $10 \mathrm{k}$ measurements have been performed between March and July 2016.

The user interface of our mobile app mimics a professional SLM and allows two different measurement modes (Fig. 4A): the manual mode (in which users can decide starting time and duration of measurement sessions) and the automatic one (in
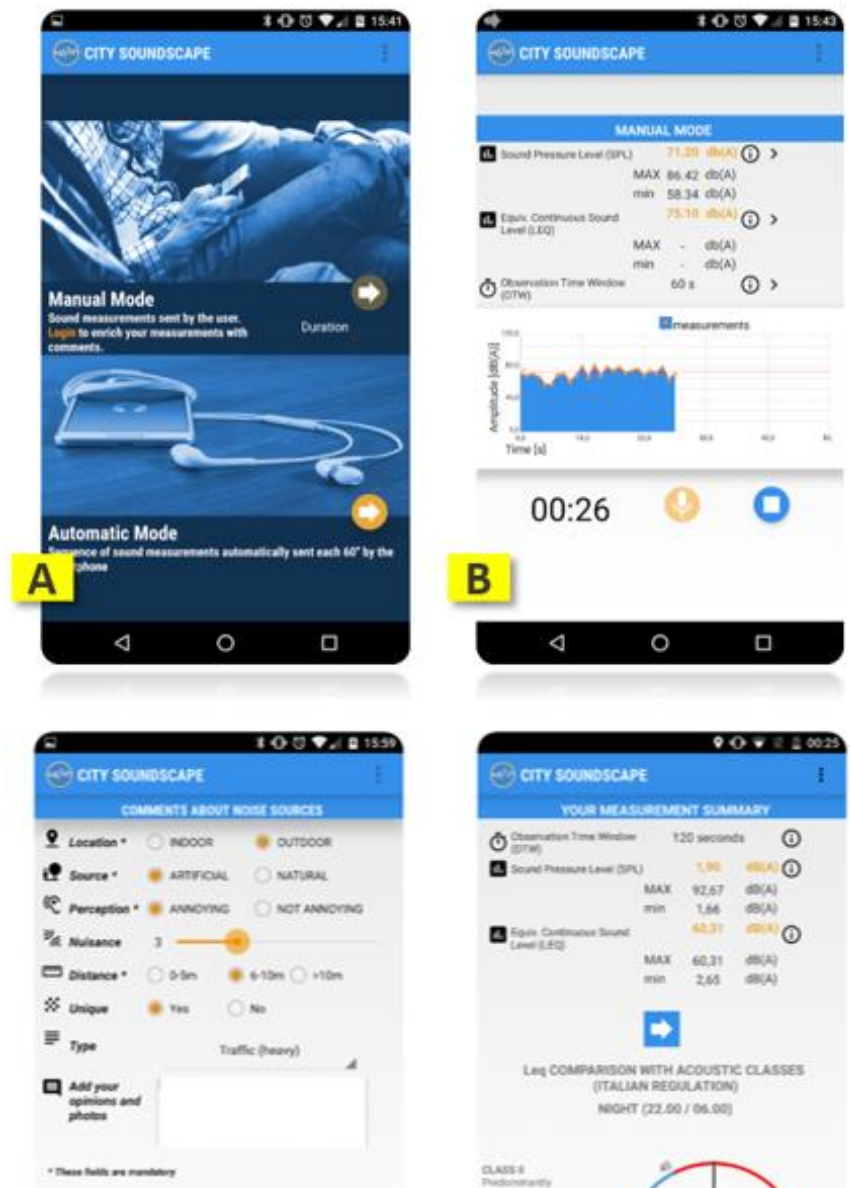

$\mathbf{0}$
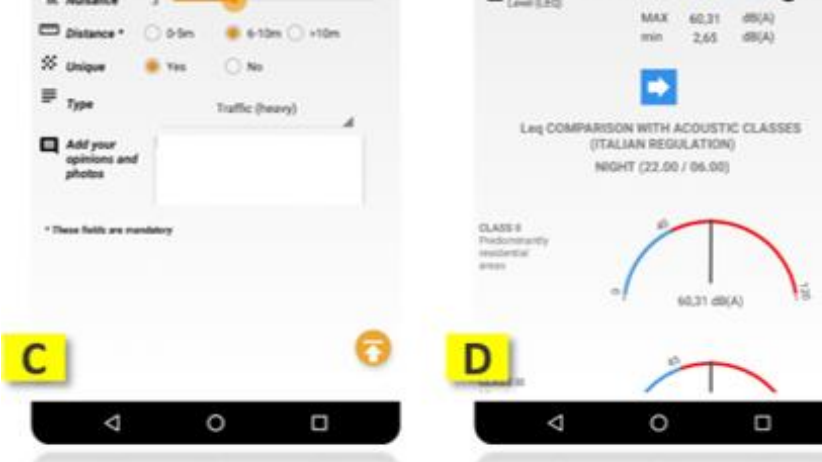

Fig. 4. Mobile application: measurement typology selection page (A); live measurement page (B); user's comment page (C); measurement summary page $(\mathrm{D})$.

which users simply start the app that will keep on collecting measurements autonomously). Both SPL (blue chart) and $L_{E Q(T)}$ (red line) values are reported and plotted on a XY graph (Fig. 4B): for each of them, current, maximum and minimum values collected during the observation time window $T$ are also reported (upper part of the app page). Users can also send comments to city managers in order to help them in evaluating citizenships' perception about noise pollution (Fig. 4C) by expressing the perceived nuisance of the surrounding environment according to a numerical psychometric scale. Other comment typologies are also available in order to allow users describing the noise sources they are close to (e.g., whether they are indoor or outdoor, artificial or natural, unique or not, etc.). These comments are optional but the opportunities disclosed by their availability are significant: whilst collected sound measurements are used to create objective noise maps (i.e., maps whose values comes from sensors), comments allows building subjective noise maps (i.e., maps where the nuisance levels are reported). This allows city managers 
locating areas where noise pollution is actually relevant or areas where citizens' perceptions exceed the true noise values. Also free-text comments are possible, so that city managers can be informed about any other noise-related issues identified by citizens. After each measurement session, a thorough summary is provided to the user (Fig. 4D), where it is also possible to visually compare measured $L_{E Q(T)}$ values against the six acoustic classes defined in the current Italian noise regulations.

\section{B. Measurement Accuracy and Data Quality}

In order to validate our measuring devices, several tests have been performed, according to the most recent trends in MCS accuracy verification for noise measurements [64]. This is needed since mobiles embed omnidirectional, non-professional microphones, specifically tailored to detect voices, instead of professional metering equipment which employ directional ambient microphones spanning over broader ranges of frequencies [65], thus potentially hindering measurement accuracy.

During the instrumental evaluation we have selected a fixedduration, steady, mid-level, broadband noise source and then we have repeatedly measured it by using different smartphone models and by using a professional, portable, calibrated, Class2 SLM (i.e., Sauter SU-130). By comparing the two measurement datasets, we have achieved an acceptable accuracy: data from mobiles were affected on average by a $\pm 5 \mathrm{~dB}$ bias, which confirms recent research works [64] and demonstrates that smartphones can be employed as preliminary sound monitoring stations.

Data quality is ensured by the implementation of several algorithms estimating user's behavior. This kind of assessment is needed since wrong behaviors determine unreliable measurements. Let us think to typical way of using smartphones during our daily life experiences: when not used for making phone calls or playing with mobile apps, smartphones are normally placed within pockets or bags. Therefore, their microphones are covered with several layers of fabric and other similar materials. If the acoustic monitoring app is still active when the smartphone is in such condition, the acoustic measurement is affected by an attenuation factor (i.e., acoustic transmission loss) which depends on the impedance and surface shape of the material against which we have the incidence of the incoming sound waves. Whilst several references are available for assessing the absorption and attenuation in angledependent incidence of known acoustic sources against multiple materials in controlled environments (e.g., reverberation chambers) [66], there are still uncertainties in determining the acoustic attenuation and transfer characteristics of clothes and fabrics in real-life conditions [67]. Laboratory tests have ascertained the effects of fabrics positioned statically between an ambient microphone and a noise source [68] but no extensive results are available for evaluating more complex conditions such as indirect and variable microphone placements, denser material composition and placements (e.g., weaves), presence of multi-layer materials with different acoustic transmission losses. Therefore, we decided to collect contextual data from the additional smartphone sensors (e.g.,
TABLE II

SUGGeSTED NOISE REDUCTION INTERVENTIONS (NRIS)

\begin{tabular}{|c|c|c|}
\hline NRI & $\begin{array}{c}\text { Expected } \\
\text { average } \\
\text { impact on } \\
L_{E Q(T)} \\
{[\mathrm{dB}(\mathrm{A})]}\end{array}$ & $\begin{array}{l}\text { Estimated } \\
\text { average cost } \\
\text { (buying }+ \\
\text { installation) }\end{array}$ \\
\hline \multicolumn{3}{|l|}{ Low-noise road pavement installation [70] } \\
\hline Two-layer porous asphalt & -5.5 & $29 € / \mathrm{m}^{2}$ \\
\hline Thin-layer asphalt & -2.5 & $22 € / \mathrm{m}^{2}$ \\
\hline Porous asphalt & -3 & $23 € / \mathrm{m}^{2}$ \\
\hline Stone mastic asphalt & -1.5 & $17 € / \mathrm{m}^{2}$ \\
\hline Dense/concrete asphalt (ref. value) & 0 & $12 € / \mathrm{m}^{2}$ \\
\hline \multicolumn{3}{|c|}{ Speed reduction (mixed traffic, normal asphalt) [71] } \\
\hline From 40 to $30 \mathrm{~km} / \mathrm{h}$ & -0.3 & $0.2 \div 20 \mathrm{k} € /$ sign \\
\hline From 50 to $40 \mathrm{~km} / \mathrm{h}$ & -1.4 & " \\
\hline From 60 to $50 \mathrm{~km} / \mathrm{h}$ & -2.1 & “ \\
\hline From 70 to $60 \mathrm{~km} / \mathrm{h}$ & -1.8 & “ \\
\hline From 80 to $70 \mathrm{~km} / \mathrm{h}$ & -1.7 & “ \\
\hline From 90 to $80 \mathrm{~km} / \mathrm{h}$ & -1.3 & “ \\
\hline \multicolumn{3}{|c|}{ Mixed traffic flow reduction (normal asphalt) [71] } \\
\hline $10 \%$ & -0.5 & $0.2 \div 20 \mathrm{k} € /$ sign \\
\hline $20 \%$ & -1.0 & “ \\
\hline $30 \%$ & -1.6 & “ \\
\hline $40 \%$ & -2.2 & “ \\
\hline $50 \%$ & -3.0 & “ \\
\hline $75 \%$ & -3.4 & “ \\
\hline \multicolumn{3}{|l|}{ Traffic calming measures [44] } \\
\hline Definition of a $30 \mathrm{~km} / \mathrm{h}$ zone & -2 & $0.2 \div 20 \mathrm{k} € /$ sign \\
\hline Installation of a roundabout & -4 & $150 \div 350 \mathrm{k} €$ \\
\hline Night-time restriction on heavy vehicles & -7 & $0.2 \div 20 \mathrm{k} € /$ sign \\
\hline Installation of round-top speed humps & -2 & $600 € / \mathrm{m}^{2}$ \\
\hline Installation of flat-top humps & +2 & $500 € / \mathrm{m}^{2}$ \\
\hline Installation of speed tables & -2.5 & $600 € / \mathrm{m}^{2}$ \\
\hline Installation of rubber speed bumps & -1 & $200 € / \mathrm{m}$ \\
\hline Installation of rumble strips & +6 & $500 € /$ set \\
\hline \multicolumn{3}{|c|}{ Noise barriers (direct interventions on noise exposure) [72], [73] } \\
\hline Acrylic, transparent (thickness: $15 \mathrm{~mm}$ ) & -20 & $550 \div 600 € / \mathrm{m}^{2}$ \\
\hline Concrete + expanded clay $(10 \mathrm{~cm})$ & -25 & $350 \div 450 € / \mathrm{m}^{2}$ \\
\hline Aluminum + insulating foam $(5 \mathrm{~mm})$ & -15 & $450 \div 500 € / \mathrm{m}^{2}$ \\
\hline Earth berm $(2 \mathrm{~m})$ & -10 & $150 \div 200 € / \mathrm{m}^{2}$ \\
\hline
\end{tabular}

light sensor, proximity sensor, 3 -axis accelerator, etc.) in order to assess, with a certain level of confidence, the position of the smartphone (e.g., in open air, in pocket/bag, partially obstructed, etc.). Measurements coming from devices which are evaluated as covered or partially obstructed are marked as less reliable than measurements coming from smartphones held in open air.

In addition, a uni-variate outlier-detection algorithm has been implemented, in order to remove measurements having an excessive sound level amplitude in a given temporal window. Moreover, when using MCS-based applications, users are typically concerned about battery consumption rates. Our prototype tests have shown no significant reduction in battery levels when using the mobile app, since only the microphone and the GPS are used.

\section{Noise Reduction Suggestion System}

Urban policy makers need ad hoc solutions for facing challenges promptly and effectively within their cities. High environmental noise levels are typically considered as a warning sign for several aspects: congestion of roads, issues in transportation systems, potential dissatisfaction of citizens and worsening of life quality or unauthorized acoustic emissions from industrial compounds. Several urban noise sources can be 
identified: vehicular/railway/air traffic, industries, production facilities, large-scale events. The proposed Web app addresses the noise generated by vehicular traffic only, since it represents the most relevant cause of urban noise pollution [69].

A series of Noise Reduction Interventions (NRIs) can be applied to decrease vehicular noise and, at the same time, to increase the transportation system quality and sustainability. These actions, however, reduce acoustic emissions differently and have different costs. Additionally, some of them are suitable to be applied along an entire road (point-NRI, e.g., road pavement, noise barriers, etc.), while some others are more effective if placed in a specific point along a road (road-NRI, e.g., speed bumps, rumble strips, etc.).

Moreover, some interventions directly impact noise sources (e.g., vehicle maximum speed reduction), whilst some others refer to noise exposure (e.g., noise barriers), thus requiring different enforcement methods. Amongst the proposed NRIs, low-noise asphalts are not expensive $\left(30 € / \mathrm{m}^{2}\right.$ on average) but very effective options for reducing traffic noise [70]. Moreover, they can be applied directly in noise hot spots without requiring environmental or architectonic modifications.

Similarly, speed limit enforcements, especially in the range $40-70 \mathrm{~km} / \mathrm{h}$, and traffic flow restriction measures are particularly useful, not only in terms of noise reduction but also for air quality and road safety [71]. Typically, such solutions have even lower costs for cities than low-noise asphalts but they may have collateral social costs due to travel time losses. Traffic calming measures (e.g., speed bumps, rumble strips, roundabouts) have been also considered but city mangers evaluate their application since each speed reduction artefact may generate additional noise (e.g., once a vehicle reaches a road hump).

Noise barriers are the most suitable solution for reducing noise exposure ([72], [73]) but their average costs are quite relevant. More specifically, noise barriers differ in their acoustic insulation and acoustic absorption properties depending on several factors: building materials, shapes, thickness, bending angles at the top of the barrier and height are just some of the technical parameters that determine the overall behavior of a barrier with respect to an incident acoustic wave. Since a detailed analysis of the technical specifications available for noise barriers is out of scope in this research context, we have selected a set of typical noise barriers normally used in road acoustic insulation in Italy. Acrylic barriers, on the one hand, are very effective, thin (up to $2 \mathrm{~cm}$ ) and have a low visual impact since normally transparent acrylic panels are used but on the other hand are very costly. Aluminum-based noise barriers are not so widely adopted even if they are lessexpensive and offer a good sound protection (a sound-insulating foam is placed between two Aluminum panels). Concrete barriers having internal cavities filled with expanded clay (which gives the sound absorption capability) have proven to be effective as well and represent one of the most-widely adopted solution. Earth berms represent a less-costly, even if less effective, alternative. They does not require any significant building intervention to be installed but they have a limited range of applications (e.g., open or rural areas).
Table II reports all the NRIs described so far. These NRIs are proposed by the Web app to city managers. Each NRI is characterized by the following set of information: expected impact on $L_{E Q(T)}$ and estimated average costs. The estimated impact on $L_{E Q(T)}$ is determined by the analysis of the domain scientific literature, whose most significant works are referenced right within the table. The average costs are estimated: 1) on a per-square-meter basis if it refers to NRI having a surface extension (such as road pavements or noise barriers; 2) on a per-item basis if it refers to specific NRIs such as rumble strips or round speed bumps; 3 ) on a per-sign basis if the enforcement of the NRI simply requires the installation of a set of road signs (e.g., for notifying a specific speed limit or a traffic restriction). The average costs are also estimated by referring to the actual pricing lists provided by some private companies and by the Italian National Highway Authority. It is worth to mention that some of the proposed NRI belong to the typical traffic calming measures; however, some of them, despite quite effective in enforcing the traffic/speed reduction increase the overall $L_{E Q(T)}$ (e.g., rumble strips).

Therefore, we offer city managers the possibility of verifying the impact of the selected NRIs on a given area, by examining the expected reduction in the overall noise level once the NRI is put in place. By doing this, city managers can also evaluate and decide to move some more effective (but louder) traffic calming measures in areas where the overall $L_{E Q(T)}$ is well beyond the limits.

Starting from such a variegated context, we have developed in [10] a Web application for city managers that allows them examining on a geo-referenced map (from OpenStreetMap) the noise measurements collected from smartphones and the effect of a specific NRI applied in a given city area. Several technicians from public authorities involved in traffic management and urban planning have validated the Web app. After the validation, several new functional requirements have been elicited, which have led to the new version of the Web app described in the remaining part of this Section. The involved testers have required the following additional features that were not implemented in [10]:

1. detailed info about each NRI in terms of both its financial and acoustic impact;

2. availability of counters enlisting the applied NRIs;

3. suitable options for downloading the measurement datasets;

4. visual summaries on the overall detected noise levels;

5. possibility to revert to the original noise maps after having applied a set of NRIs, without resetting the map.

Figure 5 depicts the new Web app interface we have designed and implemented according to the new requirements. The Web app is available for guest navigation at [74].

Once logged in, city managers can retrieve noise maps that represent a snapshot of the acoustic emissions detected in a given geographical area during a specific time-window by users having installed on their smartphones our mobile app (see Section VII.A). The ad-hoc developed Web application for this kind of stakeholders is represented in Figure 5. Each measurement point is represented as a marker, whose $L_{E Q(T)}$ 


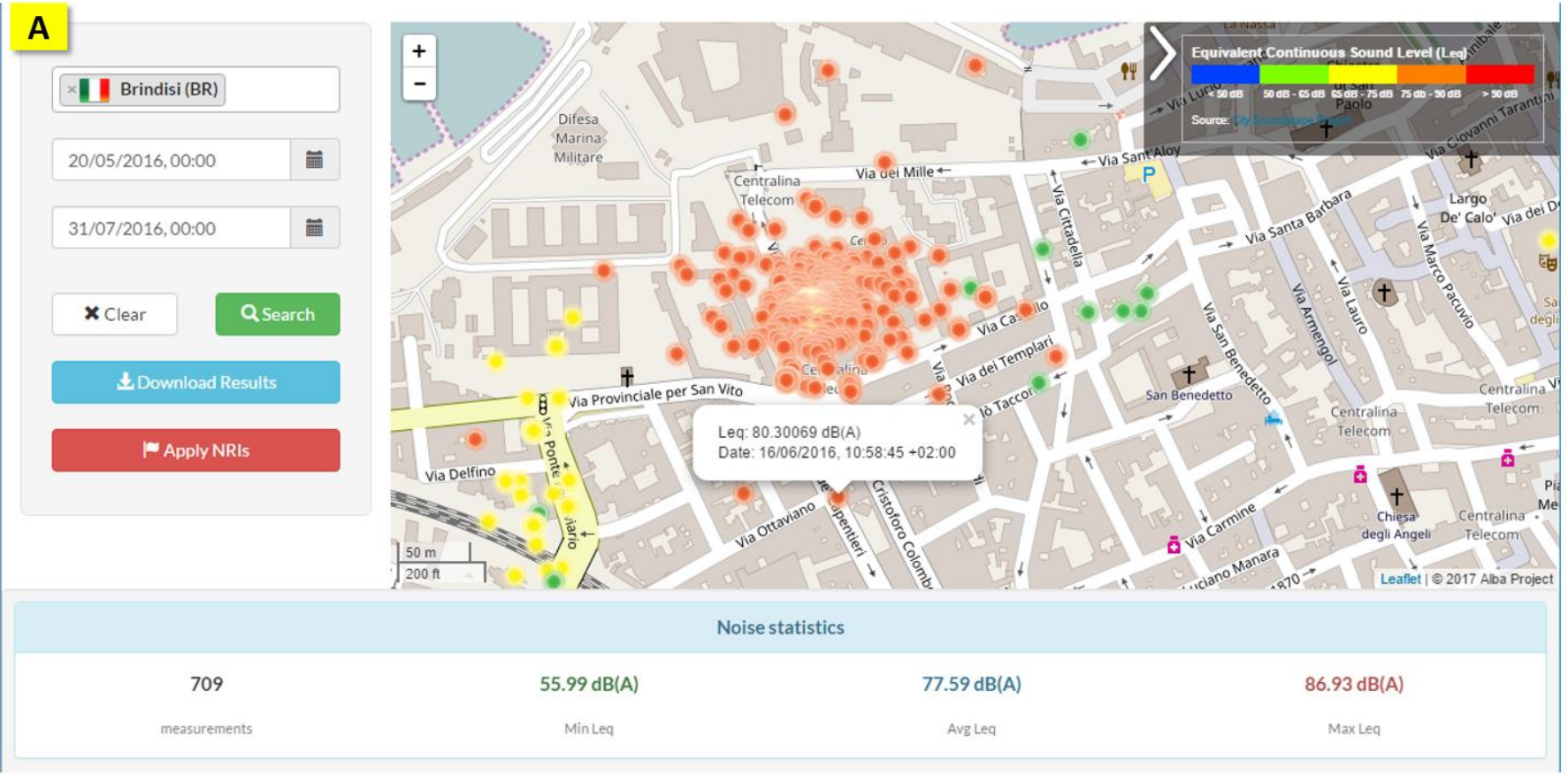

\section{B}
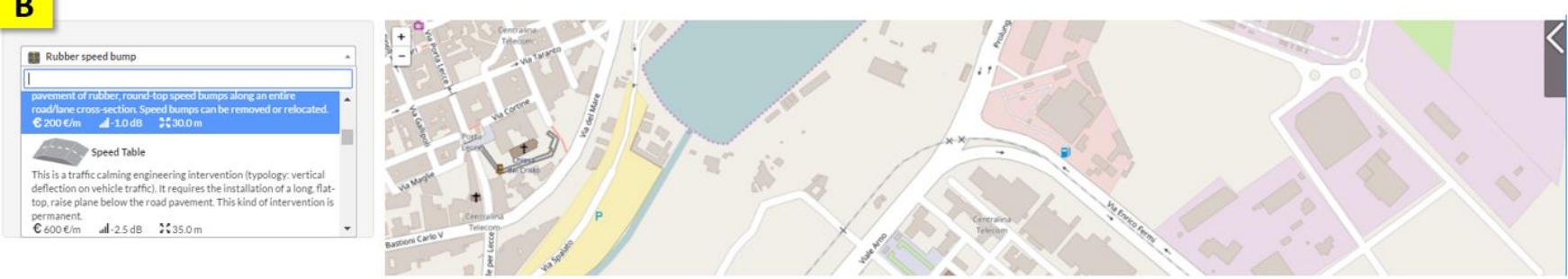

C
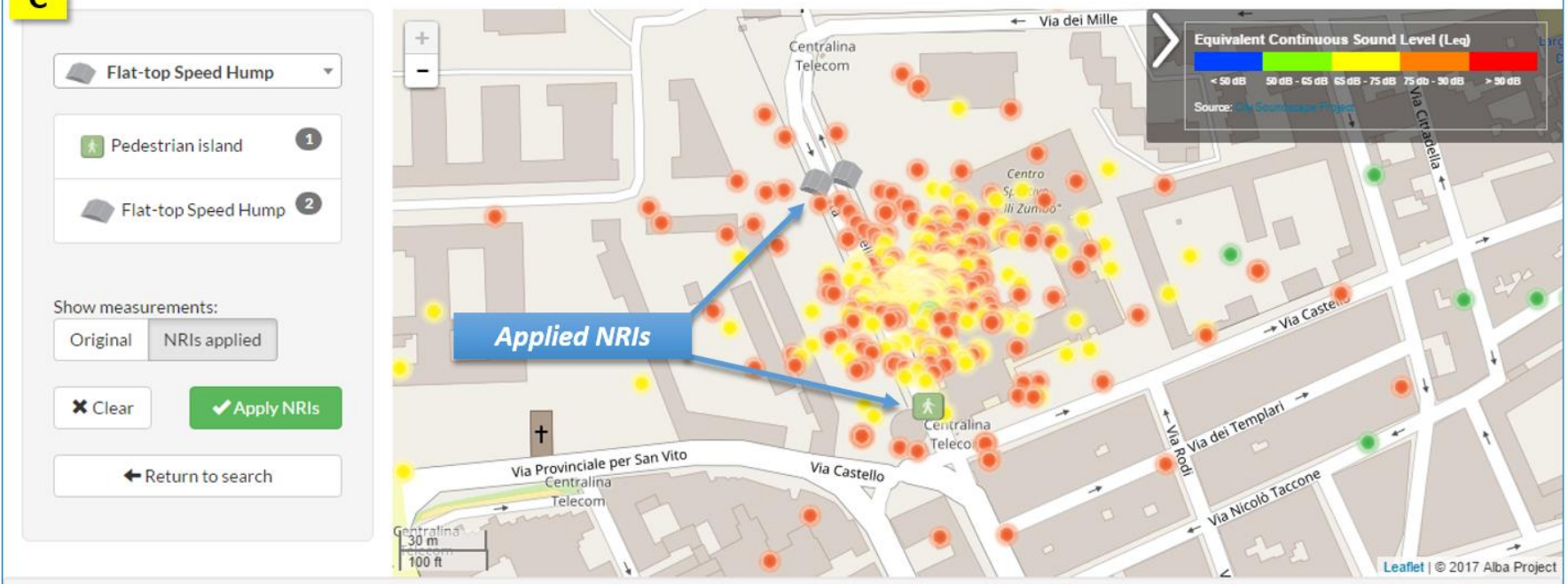

Noise statistics

$\begin{array}{lc}709 & 55.99 \mathrm{~dB}(\mathrm{~A}) \text { 55.99dB(A) } \\ \text { Min Leq } & 73.86 \mathrm{~dB}(\mathrm{~A}) \text { 77.59dB(A) }\end{array}$

$86.93 \mathrm{~dB}(\mathrm{~A}) \quad 86.93 \mathrm{~dB}(\mathrm{~A})$

Fig. 5. Web app for urban planning: noise map after dataset selection (A); NRI selection (B); NRI application and noise map re-plotting (C).

value is visualized according to a rainbow intensity scale. For value and measurement timestamp are also available (see the each measurement point, the corresponding measured $L_{E Q(T)}$ callout visible in Fig.5A). A useful summary detailing how 
many measurement points have been collected in the selected area and time-window is presented in a dedicated low horizontal frame, along with the $\mathrm{min} / \mathrm{avg} / \mathrm{max} L_{E Q(T)}$ values (see again Fig.5A). The entire dataset from which the depicted noise map has been obtained can be also downloaded as a .csv file. NRIs can be selected from a dropdown list (see Fig.5B) where they are enslisted with an icon, a brief description, the estimated cost, $L_{E Q(T)}$ reduction (according to Table II) and range of effectiveness. For instance, by referring again to Fig.5B, a speed table has an average installation cost of nearly $600 € / \mathrm{m}^{2}$ and it is expected to bring a significant $L_{E Q(T)}$ reduction of up to $2.5 \mathrm{~dB}(\mathrm{~A})$ in a circular neighborhood centered on its installation point and having a radius of up to $35 \mathrm{~m}$, without considering any additional obstacle. Then, the selected NRIs can be placed as movable objects directly on the map and enumerated (see the progressive counters shown in the left vertical framework in Fig.5B).

The "Apply NRIs" button allows re-plotting the noise map corresponding to the original geographical area and time window. Its triggered effect is depicted in Fig.5C. The $L_{E Q(T)}$ value measured at the points that fall within the application range of each NRI is recalculated, depending on the estimated $L_{E Q(T)}$ reduction provided by the considered NRIs and the noise map is plotted again accordingly on the screen. Let us consider again Fig.5A: in that figure a particularly noisy area was shown, characterized by the average $L_{E Q(T)}$ of $77.59 \mathrm{~dB}(\mathrm{~A})$. In Fig.5C, two flat-top speed humps have been placed along the main road in the Northern part of the area under examination and a pedestrian island has been placed in the southern part of the same road. As it can be seen, both visually thanks to the difference in the colored markers and numerically thanks to the summary in the low horizontal framework, the adopted NRIs could reduce the average $L_{E Q(T)}$ of approximately $4 \mathrm{~dB}(\mathrm{~A})$. The updated noise map can be switched to its original configuration (i.e., the one without the NRIs) by simply acting on a proper button in the left vertical framework. City managers can progressively check what configuration of NRIs can be the most effective by switching through the different views and by inserting and moving conveniently the NRI markers.

As a conclusive remark, in order to provide noise perception reference values, it is worth to mention that $\mathrm{a} \pm 2 \mathrm{~dB}(\mathrm{~A})$ variation is barely noticeable by humans, a $\pm 3 \mathrm{~dB}(\mathrm{~A})$ variation is perceptible, a $\pm 6 \mathrm{~dB}(\mathrm{~A})$ is clearly perceived, a $\pm 10 \mathrm{~dB}(\mathrm{~A})$ is perceived as the doubling/halving of the loudness of a given sound.

\section{CONCLUSION}

Novel technological enablers can push forward the adoption of the Smart City model in a constantly greater number of cities. Amongst these enablers, a new way of collecting sensor data has recently emerged under the definition of Mobile Crowd Sensing (MCS). It allows gathering data streams from smartphone built-in sensors in a very smooth way, since no expensive professional equipment and no skilled technician are required to manage the data collection activity. The MCS paradigm offers several benefits in terms of sufficient measurement accuracy and ease of deployment but it requires at the same time proper data management processes in order to prepare data to be effectively used. In this paper, an improved version of the MCS-based platform presented in [10] has been discussed. The platform, named City Soundscape, offers an Android-based mobile app for collecting acoustic measurements through smartphone-embedded microphones, according to two noise exposure quantifiers adopted in the noise monitoring Italian and European regulation. City Soundscape also offer a Web-based app for suggesting city managers the most adequate Noise Reduction Interventions (NRIs) for a given scenario in terms of expected noise reduction, estimated buying and installation cost, expected range of effectiveness. The mobile app has been largely tested by exploiting the collaboration of a series of schools in the province of Brindisi (Southern Italy), whose students have participated in educational activities centered on acoustics by performing several outdoor measurement campaigns. The platform architecture has been redesigned as a microservices architecture in order to exploit the advantages offered by this recent architectural approach. The Web app has been deeply revised thanks to the input provided by several city managers who have suggested a series of functional improvements to its original configuration. A more detailed analysis of the urban noise scenario and a thorough discussion on the proposed NRIs enrich this paper as well.

\section{ACKNOWLEDGMENT}

The authors also wish to thank Eng. Sergio De Lorenzis for the support provided during platform development and testing.

\section{REFERENCES}

[1] G. Reinsons, "Smart City Solutions for Small City," in Proc. of UNCTAD'15, 2015.

[2] J.-S. Hwang and Y. H. Choe, "Smart Cities Seoul : a case study," 2013.

[3] O. Benedikt, "The Valuable Citizens of Smart Cities: the Case of Songdo City,” Grad. J. Soc. Sci., vol. 12, no. 2, pp. 17-36, 2016.

[4] L. Catarinucci, M. Cappelli, and R. Colella, "a Novel Low-Cost Multisensor- Tag for Rfid Applications in," Microw. Opt. Technol. Lett., vol. 50, no. 11, pp. 2877-2881, 2008.

[5] L. Catarinucci, R. Colella, M. De Blasi, L. Patrono, and L. Tarricone, "Enhanced UHF RFID tags for drug tracing," J. Med. Syst., vol. 36, no. 6, pp. 3451-3462, 2012.

[6] L. Catarinucci, R. Colella, A. Esposito, L. Tarricone, and M. Zappatore, "A context-aware smart infrastructure based on RFID sensor-tags and its application to the health-care domain," in ETFA 2009 - 2009 IEEE Conference on Emerging Technologies and Factory Automation, 2009.

[7] S. Heggen, "Integrating Participatory Sensing and Informal Science Education," in Proc. of 2012 ACM Conf. on Ubiquitous Computing (UbiComp'12), 2012, pp. 552-555.

[8] B. Guo, Z. Yu, X. Zhou, and D. Zhang, "From participatory sensing to Mobile Crowd Sensing," in 2014 IEEE International Conference on Pervasive Computing and Communication Workshops, PERCOM WORKSHOPS 2014, 2014, pp. 593-598.

[9] S. S. Kanhere, "Participatory Sensing: Crowdsourcing Data from Mobile Smartphones in Urban Spaces," in Proc. of 12th IEEE Int. Conf. on Mobile Data Management (MDM2011), 2011, pp. $1-6$.

[10] M. Zappatore, A. Longo, and M. A. Bochicchio, "Using mobile crowd sensing for noise monitoring in smart cities," in 2016 
International Multidisciplinary Conference on Computer and Energy Science, SpliTech 2016, 2016, pp. 1-6.

[11] BSI (British Standard Institute), "Smart Cities. Vocabulary." 2014.

[12] ITU, "Focus Group on Smart Sustainable Cities," 2015.

[13] R. Kitchin, "The Real-Time City? Big Data and Smart Urbanism," GeoJournal, vol. 79, no. 1, pp. 1-14, 2014.

[14] A. Townsend, Smart cities: big data, civic hackers, and the quest for a new utopia. New York, NY, USA: W.W. Norton \& Co., 2014.

[15] R. Kitchin, "Data-Driven, Networked Urbanism,” 2015.

[16] Ericsson, "Ericsson Mobility Report 2016 - On the Pulse of the Networked Society," 2016.

[17] R. K. Ganti, "Mobile Crowdsensing: Current State and Future Challenges," IEEE Commun. Mag., vol. 49, no. 11, pp. 32-39, 2011.

[18] A. Tisma, M. de Weerdt, M. B. van Riemsdijk, M. Warnier, and R. van der Velde, "Smart Phones for a Smart City: Requirements for Context Aware Mobile Application for Landscape and Urban Planning," Proc. 14th Int. Conf. Comput. Urban Plan. Urban Manag. (CUPUM 2015), p. , 2015.

[19] S. K. Vashist, E. M. Schneider, and J. H. T. Luong, "Commercial Smartphone-based Devices and Smart Applications for Personalized Healthcare Monitoring and Management," Diagnostics, vol. 4, pp. 104-128, 2014.

[20] G. Chugh, D. Bansal, and S. Sofat, "Road Condition Detection Using Smartphone Sensors: A Survey," Int. J. Electron. Electr. Eng., vol. 7, no. 6, pp. 595-602, 2014.

[21] S. Hu, L. Su, H. Liu, H. Wang, and T. F. Abdelzaher, "Smartroad: Smartphone-based crowd sensing for traffic regulator detection and identification," ACM Trans. Sens. Networks, vol. 11, no. 4, p. 27, 2015.

[22] Beecham Research, "Smart Parking: Towards Building Smarter Cities," Cambridge, UK, 2014.

[23] C. Leonardi, A. Cappellotto, M. Caraviello, B. Lepri, and F. Antonelli, "SecondNose: an Air Quality Mobile Crowdsensing System," in Proc. of 8th Nordic Conference on HumanComputer Interaction: Fun, Fast, Foundational (NordiCHI'14), 2014, pp. 1051-1054.

[24] E. Minkman, "Citizen Science in Water Quality Monitoring," TU Delft, 2015.

[25] A. Longo, M. Zappatore, and M. A. Bochicchio, "Towards Massive Open Online Laboratories: An experience about electromagnetic crowdsensing," in Proceedings of 2015 12th International Conference on Remote Engineering and Virtual Instrumentation (REV), 2015, pp. 43-51.

[26] A. Longo, M. Zappatore, and M. A. Bochicchio, "Collaborative Learning from Mobile Crowd Sensing: a Case Study in Electromagnetic Monitoring," in 2015 IEEE Global Engineering Education Conference (EDUCON), 2015, pp. 742-750.

[27] Y. Ishigaki, Y. Matsumoto, R. Ichimiya, and K. Tanaka, "Development of Mobile Radiation Monitoring System Utilizing Smartphone and Its Field Tests in Fukushima," IEEE Sens. J., vol. 13, no. 10, pp. 3520-3526, 2013.

[28] A. Salfinger, W. Retschitzegger, W. Schwinger, and B. Proell, "CrowdSA - Towards Adaptive and Situation-driven CrowdSensing for Disaster Situation Awareness," in Proc. of 2015 IEEE Int. Inter-Disciplinary Conf. on Cognitive Methods in Situation Awareness and Decision Support (CogSIMA), 2015, pp. 1-7.

[29] J. . Radianti, J. . Dugdale, J. J. . Gonzalez, and O.-C. . Granmo, "Smartphone sensing platform for emergency management," in ISCRAM 2014 Conference Proceedings - 11th International Conference on Information Systems for Crisis Response and Management, 2014, no. May, pp. 379-383.

[30] A. Stopczynski, J. E. Larsen, S. Lehmann, L. Dynowski, and M. Fuentes, "Participatory bluetooth sensing: A method for acquiring spatio-temporal data about participant mobility and interactions at large scale events," 2013 IEEE Int. Conf. Pervasive Comput. Commun. Work. PerCom Work. 2013, pp. 242-247, 2013.

[31] Performance Audio LLC, "Decibel Meter Pro." 2012.

[32] Mobile Essentials, "Sound Meter PRO." Google Play, 2016.

[33] D. Gates, "Advanced dB (SPL) Meter." Google Play, 2016.

[34] R. K. Rana, C. T. Chou, S. S. Kanhere, N. Bulusu, and W. Hu, "Ear-Phone: An End-to-End Participatory Urban Noise Mapping System," in Proceedings of the International Conference on Information Processing in Sensor Networks IPSN, 2010, pp. 105-116.

[35] S. Leao, K. L. Ong, and A. Krezel, "2Loud?: Community mapping of exposure to traffic noise with mobile phones," in Environmental Monitoring and Assessment, 2014, vol. 186, no. 10, pp. 6193-6206.

[36] A. Bartonova, "CITI-SENSE - Citizens' Observatories and What They Can Do For You," Oslo, Finland, 2016.

[37] E. D'Hondt, M. Stevens, and A. Jacobs, "Participatory noise mapping works!," p. 27, 2011.

[38] S. Hachem, V. Mallet, R. Ventura, A. Pathak, V. Issarny, P. G. Raverdy, and R. Bhatia, "Monitoring noise pollution using the urban civics middleware," Proc. - 2015 IEEE 1st Int. Conf. Big Data Comput. Serv. Appl. BigDataService 2015, pp. 52-61, 2015.

[39] J. Kuhn and P. Vogt, "Analyzing Acoustic Phenomena with a Smartphone Microphone,” Phys. Teach., vol. 51, pp. 118-119, 2013.

[40] P. Klein, "Classical Experiments Revisited: Smartphones and Tablet PCs as Experimental Tools in Acoustics and Optics," Phys. Educ., vol. 49, 2014.

[41] G. Cardone, A. Corradi, L. Foschini, and R. Ianniello, "ParticipAct: A Large-Scale Crowdsensing Platform," IEEE Trans. Emerg. Top. Comput., vol. 4, pp. 21-32, 2016.

[42] TNS Opinion \& Social, "Attitudes of Europeans towards Urban Mobility - Special Eurobarometer," 2013.

[43] ISPRA, "Qualità dell'Ambiente Urbano," Rome, Italy, 2014.

[44] H. Bendtsen, J. Haberl, U. Sandberg, G. Watts, and E. Pucher, "Traffic Management and Noise Reducing Pavements Recommendations on Additional Noise Reducing Measures (SILVIA, Sustainable Road Surfaces for Traffic Noise Control)," Roskilde, Denmark, 2005.

[45] I. I. Bogoch, R. A. House, and I. Kudla, "Perceptions about hearing protection and noise-induced hearing loss of attendees of rock concerts," Can. J. Public Heal., vol. 96, no. 1, pp. 69-72, 2005.

[46] P. Eibich, K. Kholodilin, C. Krekel, and G. G. Wagner, "Aircraft Noise in Berlin Affects Quality of Life Even Outside the Airport Grounds," Berlin, Germany, 2015.

[47] W. Passchier-Vermeer and W. F. Passchier, "Noise exposure and public health," Env. Heal. Perspect, vol. 108, pp. 123-131, 2000.

[48] T. Hellmuth, T. Classen, R. Kim, and S. Kephalopoulos, "Methodological guidance for estimating the burden of disease from environmental noise," p. 69, 2012.

[49] C. Tiesler, M. Birk, and E. Thierling, "Exposure to road traffic noise and children's behavioural problems and sleep disturbances: results from the GINIplus and LISAplus studies," Environ. Res., vol. 123, pp. 1-8, 2013.

[50] Directive 2002/49/EC of the European Parliament and of the Council of 25 June 2002 relating to the Assessment and Management of Environmental Noise. European Parliament, 2002.

[51] European Commission, "REFIT Evaluation of the Directive 2002/49/EC relating to the Assessment and Management of Environmental Noise," Brussels, Belgium, 2016.

[52] F. Alton Everest and K. Pohlmann, Master Handbook of Acoustics, 5th ed. T. Electronics, 2009. 
[53] DPCM 14/11/1997 - Determination of Threshold Values for Acoustic Sources. 1997.

[54] LQ 26/10/1995/447 - Framework Law on Environmental Noise Pollution. Italy, 1995.

[55] DPR 30/03/2004/142 - Presidential Decree on the Noise Pollution deriving from the Vehicular Traffic. 2004.

[56] M. Golfarelli and S. Rizzi, Data Warehouse Design: Modern Principles and Methodologies, 1st ed. McGraw-Hill, 2009.

[57] FIWARE, "Orion GE Context Broker," 2016. [Online]. Available: http://catalogue.fiware.org/enablers/publishsubscribe-contextbroker-orion-context-broker. [Accessed: 01-Nov-2016].

[58] FIWARE, "FIWARE Architecture," 2014. [Online]. Available: https://forge.fiware.org/plugins/mediawiki/wiki/fiware/index.ph p/FIWARE_Architecture. [Accessed: 01-Nov-2016].

[59] Hitachi Group, "Pentaho Community Edition (CE): Data Integration, Business Analytics and Big Data," 2016. [Online]. Available: http://www.pentaho.com. [Accessed: 01-Nov-2016].

[60] M. Fowler and J. Lewis, "Microservices. A definition of this new architectural term," MartinFowler.com, 2014. [Online] Available: http://martinfowler.com/articles/microservices.html. [Accessed: 01-Nov-2016].

[61] S. Newman, Building Microservices. O'Reilly Media, 2015.

[62] J. L. Cueto and G. Licitra, "Geographic information system tools for noise mapping," in Noise Mapping in the EU: Models and Procedures, G. Licitra, Ed. London, UK: CRC Press, 2012.

[63] Google, "Google Play Store - City Soundscape," 2016. [Online]. Available:

https://play.google.com/store/apps/details?id=it.albaproject.city soundscape. [Accessed: 01-Nov-2016].

[64] C. A. Kardous and P. B. Shaw, "Evaluation of smartphone sound measurement applicationsa)," J. Acoust. Soc. Am., vol. 135, no. 4, 2014.

[65] S. H. Hawley and R. E. McClain, "Visualizing sound directivity via smartphone sensors," J. Acoust. Soc. Am., vol. 140, no. 4, pp. 2987-2995, 2016.

[66] M. Vorlander, Auralization: Fundamentals of Acoustics, Modelling, Simulation, Algorithms and Acoustic Virtual Reality. Springer Verlag, 2007.

[67] M. Van Dam, "Acoustic characteristics of the clothes used for a wearable recording device," J. Acoust. Soc. Am., vol. 136, 2014

[68] B. V. Biesel and K. A. Cunefare, "Insertion loss of personal protective clothing," Appl. Acoust., vol. 62, no. 7, pp. 831-840, 2001.

[69] EEA, "Noise in Europe 2014," 2014.

[70] W. Alberts, "Traffic Noise and Motorway Pavements," Geneva, Switzerland, 2015.

[71] L. Ellebjerg, "Noise Reduction in Urban Areas from Traffic and Driver Management - A Toolkit for City Authorities," 2008.

[72] H. Bendtsen, "Noise Barrier Design: Danish and Some European Examples," 2010.

[73] R. C. Theberge, "Comparison and Testing of Various Noise Wall Materials," Ohio University, 2015.

[74] AlbaProject s.r.l., "City Soundscape - Noise Map Visualizer," 2016. [Online]. Available: http://dss.citysoundscape.it/. [Accessed: 01-Nov-2016].

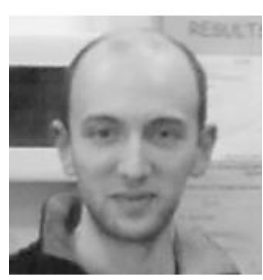

Eng. Marco Zappatore, Ph.D., is a post-doc research fellow at the Department of Innovation Engineering, University of Salento (Italy). He got a Master Degree in Communication Engineering and a Ph.D. in Information Engineering from the same university. He is with the Software Engineering \& Telemedia Lab (SET-Lab) at the same University. His main research interests are focused on Data and Knowledge management, Mobile Crowdsensing, Semantic Web and new learning paradigms. He has also expertise in electromagnetic field measurements, radio-propagation analysis and wireless communication system planning and optimization. He has co-authored more than 50 papers for national and international conferences and journals.

Prof. Antonella Longo, Ph.D., holds a degree in Computer Engineering and a Ph.D. in

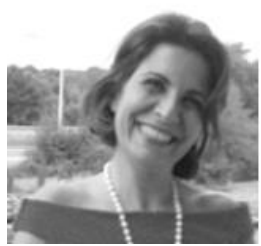
Information Engineering. Currently she is an assistant professor at the Department of Engineering for Innovation, University of Salento (Lecce), Italy, where she works in SetLab. Her research experience has been centered on the conceptual modelling of data in complex organizations and for the past 10 years she has been doing research in the service domain and conceptual modelling, publishing more the 30 publications in the field. Her main research activities concern the modelling of Service Level Agreements (SLA) in service contract management. She serves in several conferences and journal of service computing and information system as a reviewer. In her professional experience she has often mixed industrial and academic experience, in order to investigate actual problems with research and innovative approaches. Therefore, she has been involved in technology transfer projects in sectors such as Transportation, Service Governance, Public Services. Currently she is interested in the data modelling and management of smartcities governance, with specific focus on mobility and environmental scenarios.

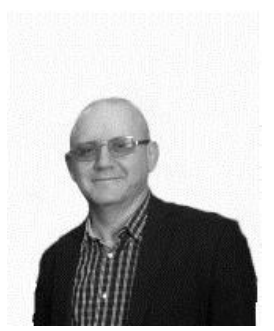

Prof. Mario A. Bochicchio, Ph.D., is an associate professor of Database at the Department of Innovation Engineering, University of Salento (Italy). He has more than 16-year experience in technology enhanced learning, in applied informatics and in databases; more than 20-year experience in teaching informatics, databases, information systems, conceptual modeling, remote engineering, online labs, more than 16 year-experience in project based learning with undergraduate and graduate students; more than 10-year experience in strategic planning, developing, implementation and evaluation of curricula in higher education on undergraduate and graduate level. He has been and is currently involved in more than 15 EU and national projects in eLearning as project leader or as project collaborator. He is author of more than 75 publications in national and international conferences and journals. 